\title{
Myxoedema Coma: A Very Rare Presentation of Severe Hypothyroidism
}

\author{
Mohamed Abdusalam Lklouk ${ }^{\mathrm{a}, \mathrm{b}}$
}

\begin{abstract}
Myxoedema coma is a rare and life threatening medical emergency with a high mortality rate. It results from severe hypothyroidism and affects almost every organ system. The salient features of myxoedema coma are decreased mental status and hypothermia, but other characteristics of severe hypothyroidism are usually present including bradycardia, hypotension, hypoglycemia and hypoventilation. Abnormal accumulation of mucin in skin and other tissues can lead to typical clinical features, for example nonpitting oedema, swollen lips, enlarged tongue and thickened nose. Diagnosis is challenging due to the paucity of cases and possibly earlier recognition of hypothyroidism as a result of improved availability of thyrotropin (TSH) assays. Clinicians can be mislead by the misnomer "coma" as patients may present with signs of cognitive impairment such as agitation, confusion and disorientation. A high index of suspicions is needed among clinicians in order to rapidly recognize the condition and make an early diagnosis. Treatment should be commenced on clinical grounds while waiting for laboratory results. It is also vital that these patients receive intensive care level treatment with close monitoring of their cardiovascular parameters and level of consciousness. In addition, severe hypothyroidism can decompensate to myxoedema coma as a result of precipitating factors, for example cold exposure, alcohol, sedative drugs, sepsis or myocardial infarction. Therefore, it is important to address and try to reverse any of these precipitants.
\end{abstract}

Keywords: Myxoedema; Hypothyroidism; Hypothermia; Thyroid stimulating hormone (TSH); Thyroxine (T4); Liothyronine

Manuscript accepted for publication September 23, 2013

${ }^{a}$ Intensive Care Unit, Department of Anaesthetics and Critical Care, University Hospital of North Tees, Hardwick Road, Stockton-on-Tees, TS198PE, United Kingdom

${ }^{\mathrm{b}}$ Corresponding address: Mohamed Abdusalam Lklouk, 6 Wentworth Lodge, 3 Wentworth Terrace, Wakefield, WF13QF, United Kingdom. Email: Mohamed.Lklouk@midyorks.nhs.uk

doi: http://dx.doi.org/10.4021/jmc1503w

\section{Introduction}

Myxoedema coma is a very rare endocrine emergency. Its recognition can be easily missed nowadays as its incidence has significantly declined in developed countries due to improved diagnosis and treatment. It is uncommon for patients to present in coma, but they manifest milder forms of altered consciousness [1]. Conversely, psychotic features may predominate in other patients; such presentation has been called myxoedema madness [2]. The diagnosis should be considered in any patient with a decreased level of consciousness who also has hypothermia, hyponatraemia and/or hypercapnoea [1]. Treatment of this condition should be early and aggressive as its mortality rate remains high at $30-40 \%$ [3-5]. Elderly patients and those with cardiovascular compromise, reduced consciousness, persistent hypothermia and sepsis are at highest risk and generally have a poorer outcome $[4$, 6]. Thyroid hormone therapy is the mainstay of treatment in addition to supportive measures and management of coexisting or precipitating problems. Glucocorticoids must be given to the patient in stress doses until adrenal insufficiency has been excluded. Here I report a case of myxoedema coma in a 60-year-old patient who presented with confusion and agitation which was initially attributed to excessive alcohol intake. Despite presenting with typical clinical signs of severe hypothyroidism, the diagnosis remained unclear in the emergency department but was picked up later by the critical care team and confirmed by laboratory tests of thyroid function. This could be possibly either due to unfamiliarity with this rare form of severe hypothyroidism or the coexistence of other confounding morbidities, for example excessive alcohol intake.

\section{Case Report}

A 60-year-old male patient was admitted to the Accident and Emergency department via ambulance. The patient's friend, who noticed that he could not talk and was not his usual character, found him at home on the floor. Neither past medical history nor hospital admissions were reported, other than 


\title{
Table 1. Salient Clinical Features of Myxoedema Coma
}

\author{
Altered level of consciousness \\ Bradycardia \\ Hypothermia \\ Hypoglycemia \\ Hypotension \\ Hyponatraemia \\ Concurrent (precipitating) illness
}

being described as an alcoholic. Empty bottles of liquor were found on scene.

On examination, he was spontaneously breathing and maintaining an oxygen saturation of about $95-97 \%$. His heart rate was around 30-32 beats/minute, with a blood pressure recording of 140/70 mm Hg. The body temperature (tympanic) was not recordable initially; an hour later, the rectal temperature was $27.4^{\circ} \mathrm{C}$. The peripheries were very cold to touch, with a capillary refill of more than 4 seconds. He was very confused, agitated (GCS 14) and deaf.

Other remarkable features were macroglossia, prognathism, puffy eyelids, thick lips, erythema ab igne over the right knee, pitting oedema over both legs and slow relaxing reflexes.

\section{Investigations}

Repeated 12 lead ECG strips showed sinus bradycardia, a chest X-ray showed left basal atelectasis and possibly a small pleural effusion.

Transthoracic echocardiography showed no pericardial effusion, left ventricular systolic function moderately impaired, right ventricular systolic function severely impaired. Laboratory blood tests showed the following: TSH (thyroid stimulating hormone) $227 \mathrm{mU} / \mathrm{L}$, FT4 (free T4) $<3 \mathrm{pmol} / \mathrm{L}$, cortisol $297 \mathrm{nmol} / \mathrm{L}, \mathrm{CK}$ (creatine kinase) 14,664 IU/L, capillary blood glucose $3.0 \mathrm{mmol} / \mathrm{L}$.

\section{Management in ICU}

Thyroxine (200 mcg), liothyronine (20 mcg) and hydrocortisone $(200 \mathrm{mg})$ were all given intravenously. Further IV liothyronine $(10 \mathrm{mcg} / 3$ daily doses) was continued for 48 hours. He was also started on regular oral L-ThyroxineT4, and regular IV hydrocortisone.

Passive re-warming was applied, and the patient's body temperature steadily increased to $35{ }^{\circ} \mathrm{C}$ over a period of about 10 hours.

Ten percent dextrose IV boluses of $100 \mathrm{~mL}$ were given to correct hypoglycemia. Invasive lines were inserted (cen- tral venous line, arterial line). His blood pressure dropped to $80 / 50 \mathrm{~mm} \mathrm{Hg}$ after starting treatment, and this was managed by IV colloid boluses, Hartmann's solution for maintenance and vasopressors (noradrenaline). Cardiac output monitoring (PiCCO - Pulse Contour Continuous Cardiac Output) showed reduced contractility and an adrenaline infusion eventually replaced the noradrenaline. The urine output was below $20 \mathrm{~mL} / \mathrm{hr}$. Inotropic support was weaned and completely stopped by the end of the second day of admission as his blood pressure, urine output and other cardiac parameters were restored within normal limits.

The patient's initial serum creatinine was $100 \mu \mathrm{mol} / \mathrm{L}$, and it peaked to $143 \mu \mathrm{mol} / \mathrm{L}$ on the second day. The serum $\mathrm{CK}$ also increased to $15,218 \mathrm{IU} / \mathrm{L}$. These figures suggested acute kidney injury secondary to rhabdomyolysis. However, aggressive IV fluid therapy and inotropic support seemed to have reversed this trend of worsening renal function and renal replacement therapy was not needed.

He was able to maintain his airway throughout, not requiring any respiratory support other than $2-4 \mathrm{~L} / \mathrm{min}$ of oxygen via nasal cannulae. The arterial blood gases persistently showed respiratory alkalosis with acceptable oxygen and carbon dioxide tensions.

$\mathrm{He}$ was confused and agitated throughout admission, which could have been aggravated by alcohol withdrawal. Haloperidol and chlordiazepoxide were given as needed. Vitamin B complex treatment was commenced as well. He was discharged to the medical ward under the care of the endocrinology team uneventfully.

\section{Discussion}

Myxoedema coma can be a result of any of the well known causes of primary hypothyroidism, for example autoimmune thyroiditis and post surgical hypothyroidism. It can also be a result of pituitary failure, and there are case reports of its association with lithium or amiodarone toxicity [7-9]. Multiple organ systems and metabolic pathways of the body are all slowed in severe long-standing hypothyroidism. The clini- 
Table 2. Management of Myxoedema Coma

\author{
Serum for TSH, T4 and cortisol \\ Switch to oral rhyroxine when patient stable \\ Stress dose intravenous hydrocortisone \\ Supportive measures \\ Ventilatory support \\ Fluids/vasopressors to correct hypotension \\ Passive rewarming \\ Correction of hypoglycaemia \\ Treatment of underlying infection
}

Administration of intravenous thyroxine (T4) and triiodothyronine (T3)

cal features of myxoedema coma are summarized in Table 1. This particular patient appeared to have almost all characteristic clinical signs of Hypothyroidism. However, many hypothyroid patients might appear normal or may have very subtle changes. It is therefore very important to be highly suspicious of myxoedema coma in any patient presenting with an altered level of consciousness plus hypothermia, bradycardia and/or hypoglycaemia.

Hypothermia is secondary to reduced metabolism leading to decreased thermogenesis, and the lower the temperature, the higher the mortality. The infrared thermometer used for this patient did not register any reading initially, and this is the case with many automated thermometers in terms of their inability to record severely hypothermic body temperatures. Therefore, the thermometer should always be checked to avoid inaccuracies. Active rewarming can cause vasodilatation and lead to more hypotension, and for this reason passive rewarming with blankets was applied.

Hypoglycaemia may be a feature of hypothyroidism, but it is usually due to coexisting adrenal insufficiency. It was assumed that this case's CK was high due to rhabdomyolysis, but a high serum lactate dehydrogenase (LDH) and creatine kinase can be biochemical features of severe hypothyroidism [10].

Severe hypothyroidism also leads to several cardiovascular effects including bradycardia, decreased myocardial contractility, a low cardiac output and hypotension [11]. This patient had a severe bradycardia and was hypotensive. Cardiac output monitoring showed reduced contractility rather than vasodilatation which is consistent with hypothyroidism. All of these cardiac changes are reversible with thyroid hormone replacement [12].

Respiratory depression can lead to hypoventilation and respiratory acidosis. In addition, macroglossia or myxoede- ma of the larynx can lead to airway obstruction. Ventilatory support is needed in some patients, and full recovery from respiratory depression can take up to three to six months following treatment [1]. The involvement of many organ systems necessitates managing myxoedema coma patients in an intensive care setting.

Blood should be drawn for measurements of serum $\mathrm{TSH}$, free T4 and cortisol prior to administration of thyroid hormone and a glucocorticoid treatment. If laboratory confirmation of hypothyroidism is delayed, treatment should be started immediately based on clinical grounds without waiting for the results. This patient's serum TSH was high and his free T4 was very low, which indicates primary hypothyroidism rather than pituitary dysfunction.

The ultimate treatment of this case was by administering a combination of intravenous thyroxine (T4), liothyronine (chemically nearly identical to triiodothyronine - T3) in addition to intravenous hydrocortisone. The biologic activity of liothyronine is greater and has a faster onset of action than T4.

Due to the rarity of the condition, not enough clinical trials have been conducted to look at different treatment regimens and outcomes. Optimum thyroid hormone therapy in myxoedema coma still remains a controversial area, and although rapidly increasing thyroid hormone levels may precipitate myocardial infarction and atrial fibrillation, this risk must be accepted as the mortality of myxoedema coma is very high if not treated.

Three different regimens have been suggested [5]: 1) intravenous or oral $\mathrm{T} 4,2$ ) intravenous $\mathrm{T} 3$ or 3 ) a combination of T4 and T3. Giving T4 intravenously has been advocated because absorption via the oral route is variable and unpredictable [13]. However, one study [14] showed that the clinical response obtained by oral $\mathrm{T} 4$ occurred promptly even in 
a case of myxoedema ileus.

We preferred to give both hormones because $\mathrm{T} 3$ has a more rapid onset of action than T4. Furthermore, peripheral conversion of $\mathrm{T} 4$ to $\mathrm{T} 3$ is impaired in hypothyroidism and in coexisting non-thyroidal illness, for example infection. But cautious dosing of $\mathrm{T} 3$ is important as high serum T3 levels have been associated with mortality [3].

When this patient became stable, Oral T4 was started and T3 was stopped.

In summary, myxoedema coma is a medical emergency that has a high mortality rate. Early diagnosis and prompt thyroid hormone and glucocorticoid administration is the cornerstone of treatment even when laboratory results are not available to confirm hypothyroidism. Supportive measures are of paramount importance in the treatment of this life threatening illness. These include treatment in the intensive care unit, ventilatory and cardiovascular support, passive rewarming, correction of hypoglycemia/electrolyte imbalance and treatment of any concurrent infection (Table 2).

\section{References}

1. Kwaku MP, Burman KD. Myxedema coma. J Intensive Care Med. 2007;22(4):224-231.

2. Westphal SA. Unusual presentations of hypothyroidism. Am J Med Sci. 1997;314(5):333-337.

3. Hylander B, Rosenqvist U. Treatment of myxoedema coma--factors associated with fatal outcome. Acta Endocrinol (Copenh). 1985;108(1):65-71.

4. Dutta P, Bhansali A, Masoodi SR, Bhadada S, Sharma N, Rajput R. Predictors of outcome in myxoedema coma: a study from a tertiary care centre. Crit Care.
2008;12(1):R1.

5. Beynon J, Akhtar S, Kearney T. Predictors of outcome in myxoedema coma. Crit Care. 2008;12(1):111.

6. Yamamoto T, Fukuyama J, Fujiyoshi A. Factors associated with mortality of myxedema coma: report of eight cases and literature survey. Thyroid. 1999;9(12):11671174.

7. Santiago R, Rashkin MC. Lithium toxicity and myxedema coma in an elderly woman. J Emerg Med. 1990;8(1):63-66.

8. Waldman SA, Park D. Myxedema coma associated with lithium therapy. Am J Med. 1989;87(3):355-356.

9. Mazonson PD, Williams ML, Cantley LK, Dalldorf FG, Utiger RD, Foster JR. Myxedema coma during long-term amiodarone therapy. Am J Med. 1984;77(4):751-754.

10. Benvenga S, Squadrito S, Saporito F, Cimino A, Arrigo F, Trimarchi F. Myxedema coma of both primary and secondary origin, with non-classic presentation and extremely elevated creatine kinase. Horm Metab Res. 2000;32(9):364-366.

11. Klein I. Thyroid hormone and the cardiovascular system. Am J Med. 1990;88(6):631-637.

12. Shenoy MM, Goldman JM. Hypothyroid cardiomyopathy: echocardiographic documentation of reversibility. Am J Med Sci. 1987;294(1):1-9.

13. Holvey DN, Goodner CJ, Nicoloff JT, Dowling JT. Treatment of Myxedema Coma with Intravenous Thyroxine. Arch Intern Med. 1964;113:89-96.

14. Arlot S, Debussche X, Lalau JD, Mesmacque A, Tolani M, Quichaud J, Fournier A. Myxoedema coma: response of thyroid hormones with oral and intravenous high-dose L-thyroxine treatment. Intensive Care Med. 1991;17(1):16-18. 